\title{
Study of Two-Dimensional Photonic Crystal Arrays for Beam Splitters
}

\author{
Gwomei WU*, Ni Ting JU, Tsung Wen CHANG, Wu Shiung FENG \\ Institute of Electro-Optical Engineering, Department of Electronic Engineering, Chang Gung University, Kweisan, Taoyuan \\ 333, Taiwan \\ cross $^{\text {ref }}$ http://dx.doi.org/10.5755/j01.ms.22.2.12928
}

\author{
Received 20 August 2015; accepted 24 January 2016
}

\begin{abstract}
The effects of periodic modulation in dielectric constants on the formation of photonic band gaps of two-dimensional arrays have been characterized. When a defect is introduced into a perfect photonic crystal, a localized effect may be found at frequencies within the photonic band gap. A line defect thus acts as a waveguide, and a point defect can behave as a resonant cavity. In this report, we investigated photonic crystal structures based on germanium $(n=4.0)$, silicon $(n=3.49)$, titanium oxide $(n=2.71)$, and silica $(n=1.50)$ materials. Both square lattice arrays and triangular lattice arrays have been studied. The two-dimensional photonic structures exhibited the Mach-Zehnder interferometer function that could be applied to optical switches or optical integrators. The beam splitter had the crossed linear defects shape, constructed by two orthogonal line defects. The ratio of defect radius to the lattice constant was 0.28 , and the best moving distance has been 0.76 to achieve equal output energy.

Keywords: photonic crystal, band gap, defect, beam splitter.
\end{abstract}

\section{INTRODUCTION}

Photonic crystals have been scientifically defined as periodically spaced dielectric structures that exhibit characteristic optical band gaps where light cannot propagate $[1,2]$. The periodic modulation of the dielectric constants can be designed to lead to the formation of useful photonic band gaps for different applications. The most common structures are nano-sized air holes in dielectric slabs and also dielectric nano-rods surrounded by lower dielectric media such as air. In the last decade, this new class of crystal structures has attracted engineering interest owing to their capabilities in controlling light emission and the propagation of light waves [3]. Wu et al. proposed a compact optical switch for self-collimated light beams in a liquid-crystal-modulated photonic crystal [4]. The optical switch was designed to work at $1550 \mathrm{~nm}$ for possible applications in photonic integrated circuits. Many researchers have been searching for the potential applications in optoelectronics and information processing, such as vertical silicon micro pillar radial junction solar cells [5], channel drop filters [6], photonic crystal fiber [7], and quantum-dot embedded interferometer [8]. Some organic and polymer systems also showed interesting results for possible applications $[9,10]$. Also, when a defect is introduced into a perfect photonic crystal system, a localized mode may become available at frequencies within the band gap.

A line defect can act as a waveguide while a point defect can be used as a resonant cavity. Thus, a two-dimensional analysis of the photonic crystal waveguides can be theoretically realized, based on the introduction of a line defect in the photonic crystal material system [11 - 13]. On the other hand, a channel add or drop filtering device can be designed by using point or line defects in a two-dimensional photonic crystal slab for a wavelength division multiplexing (WDM) system [14, 15]. The light in the waveguide can be further bent to a large angle at $90^{\circ}$ or even higherby choosing the appropriate defect lines $[16,17]$.

By combining bent waveguides of photonic crystals, it becomes possible to design beam-splitters, including $\mathrm{Y}$ form or even T-form splitters [18-21]. The devices can be used to divide the light energy into two branches, equally or not equally, depending on the purposes of the applications. The other type of beam-splitter is in the crossed linear defect shape. It may be constructed by two orthogonal line defects that divide one source of light energy into three or more branches. The more complicated beam-splitters can include two input ports and two output ports. The structures then actually act as Mach-Zehnder interferometers when one or more point defects are inserted into the crossed linear defects.

In this study, two-dimensional photonic crystal band gap structures have been analyzed based on germanium (refractive index $n=4.0)$, silicon $(n=3.49)$, titanium oxide $(n=2.71)$, and silica $(n=1.50)$ materials. Both square lattice arrays and triangular lattice arrays have been studied. The structures can be either air holes in dielectric slabs or dielectric pillars that are surrounded by air. Beam-splitter structures were further designed to include two input ports and two output ports in a square lattice array arrangement. The beam-splitter had crossed linear defects that were constituted by two missing orthogonal rows. The photonic responses in terms of output power with respect to the defect size, location, index or shape have been investigated.

\section{MODELING DETAILS}

Index guiding is the main guiding mechanism for waveguides formed by photonic crystals where some kinds of dielectric defects have been involved. However, these

\footnotetext{
* Corresponding author. Tel: +886-3211-8800; fax: +886-3211-8668.

E-mail address: wu@mail.cgu.edu.tw (G. Wu)
} 
waveguides present a change in periodicity, which must be accounted for the reflection and also transmission properties. With the theories based on the finite element analysis method, we employed the Finite-difference timedomain (FDTD) methods developed by RSoft's BandSOLVE and FullWAVE to investigate the behavior of photons [22, 23]. The simulation engine employs the plane wave expansion (PWE) algorithm. 2D method is used. The used input excitation field is $\mathrm{cw}$. It is originated from the preliminary resolution of a scattering phenomenon in the space domain and the time domain. The harmonic solutions were obtained in a second stage by a Fourier transformation in time. Both the electric field $(E)$ and the magnetic field $(H)$ components must have satisfied the time-dependent Maxwell's equations. The master equations are shown as:

$\frac{1}{\varepsilon(r)} \nabla \times\left(\nabla \times E(r)=\frac{w^{2}}{c^{2}} E(r) ;\right.$

$\nabla \times\left[\frac{1}{\varepsilon(r)} \nabla \times H(r)\right]=\left[\frac{w}{c}\right]^{2} H(r)$,

where $\varepsilon(r)$ is dielectric constant; $\omega$ is frequency of electromagnetic wave; $c$ is light velocity in vacuum. The photonic band structure can be calculated under $E$ or $H$ polarization according to the dielectric constant arrangement [24]. The dispersion of electromagnetic wave is then calculated repeatedly with convergence by transferring into Yee cells with the separation of time and space domains. The implementation of the FDTD method could be separated into the following three stages. In the first stage, the wavefront of the incident field has not reached the discretized domain, and the total field was still zero at any point. In the second or illumination stage, the equations at the boundary were introduced between the scattered field and the total field. In the final stage, also known as the relaxation stage, the incidentfield would have moved beyond the domain, and the equations became homogeneous [11,21].

\section{RESULTS AND DISCUSSION}

Fig. 1 a shows the band gap diagram for a twodimensional triangular lattice array based on silica pillars in the air. The illumination is vertical in its direction. The refractive index of silica is lower at about 1.50. The silica pillar size was varied between $0.1-0.4 \mathrm{a}$, where "a" is the lattice constant of the photonic crystal. The frequency $\omega$ is expressed in a dimensionless value after being converted with the speed of light $\mathrm{c}$ and lattice constant. The normalization of radius ratio $(r / a)$ has been also used in the figure. Most of the optical polymeric organic materials have similar refractive index values and would have shown similar behavior. Since the refractive index is not much higher than that of air, we could only find limited transverse electric (TE) mode band gaps in the triangular lattice array. There were no band gap found in the square silica lattice array. However, when a larger refractive index material was used, such as $\mathrm{TiO}_{2}$ with $n=2.71$, more optical band gaps would have become available. There are several polymorphs available for titania films [25]. The tetragonal rutile has been used for this analysis. Its optoelectronic properties can be varied by structures and processing conditions. The refractive index is also dependent upon measuring wavelength. The band gap diagram for the two- dimensional square $\mathrm{TiO}_{2}$ pillar lattice array is displayed in Fig. $1 \mathrm{~b}$.

The band gap diagram for a two-dimensional square lattice array using the highest refractive index germanium pillars $(n=4.5)$ in the air is shown in Fig. $1 \mathrm{c}$.
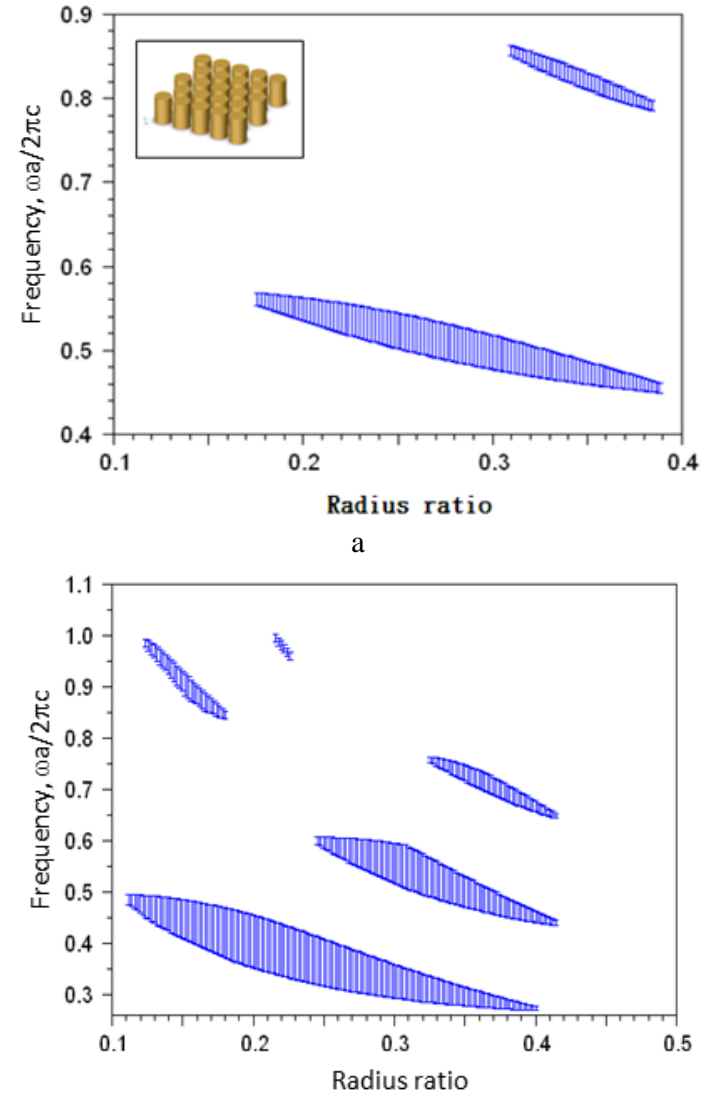

b

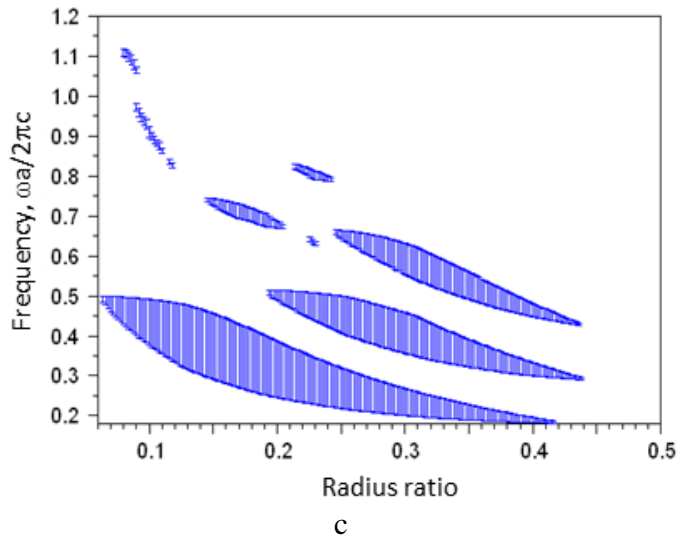

Fig. 1. a-band gap diagram for a two-dimensional triangular lattice array based on silica pillars $(n=1.50)$; only few TE mode band gaps could be found; $b$ - band gap diagram for the two-dimensional $\mathrm{TiO}_{2}$ pillar square lattice array ( $n=2.71)$; more optical band gaps have become available; $\mathrm{c}$-band gap diagram for a square lattice array using high index germanium pillars $(n=4.50)$

The much wider frequency range to exhibit band gaps could be attributed to the large difference in the refractive indices between germanium and air. It even exhibited both the transverse electric (TE) and the transverse magnetic (TM) band gaps in the air-hole-in-slab triangular lattice array. It provides an interesting array structure for some optoelectronic devices. 
Fig. 2 displays the band gap diagram for a twodimensional square lattice array based on silicon pillars. The refractive index of silicon is medium at 3.49. It is still high enough to provide band gaps in the transverse electric (TE) mode. The band gaps have been more limited for the airhole-in-slab silicon square lattice array (Fig. 3).

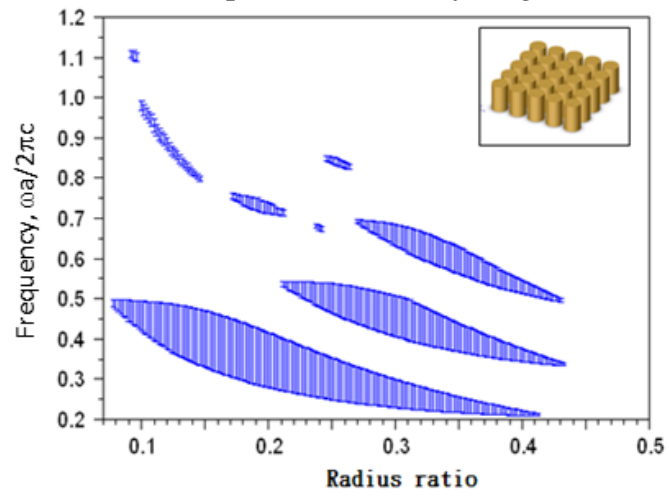

Fig. 2. Band gap diagram for a two-dimensional square lattice array based on silicon pillars

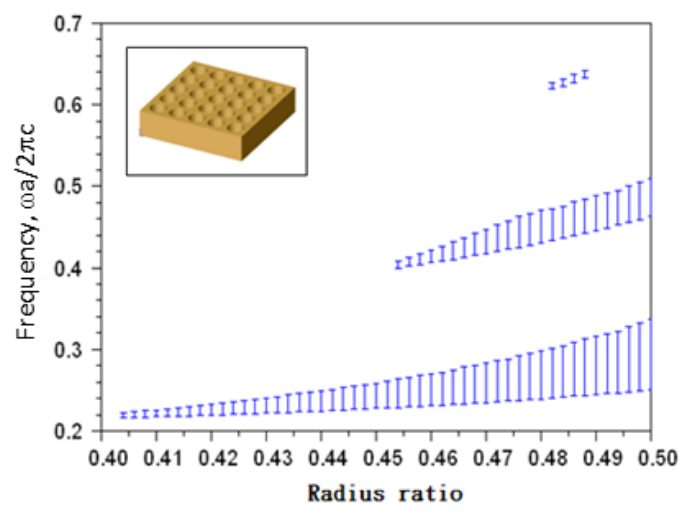

Fig. 3. Band gap diagram for a two-dimensional square lattice array based on air holes in silicon slab

On the other hand, for the triangular lattice array system, the band gaps exist well in both the transverse electric (TE) and the transverse magnetic (TM) modes. Fig. 4 shows the band gap diagram for the triangular lattice array structure from silicon pillars in the air.

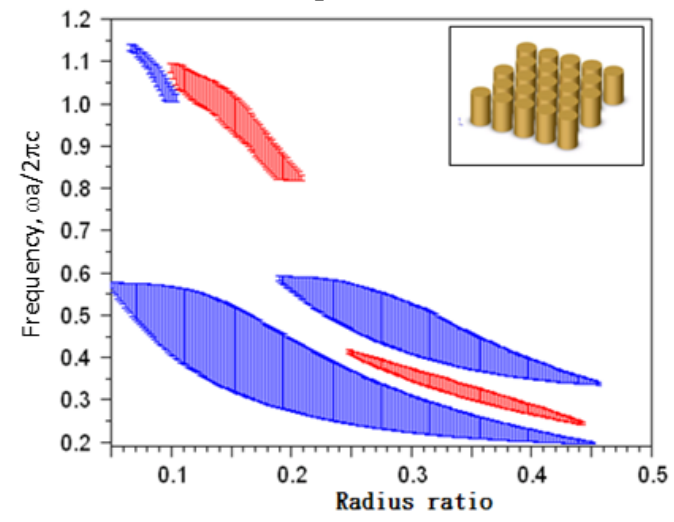

Fig. 4. Band gap diagram for a two-dimensional triangular lattice array from silicon pillars in the air. Both TE (in blue) and TM (in red) mode band gaps were observed

The periodic dielectric modulation, along with the adequate refractive index difference $(\Delta n=2.49)$, allows wide band gaps. Also, the complete TE and TM band gaps could be easily identified in the band gap diagram for a twodimensional triangular lattice array based on air holes in a silicon slab, as shown in Fig. 5.

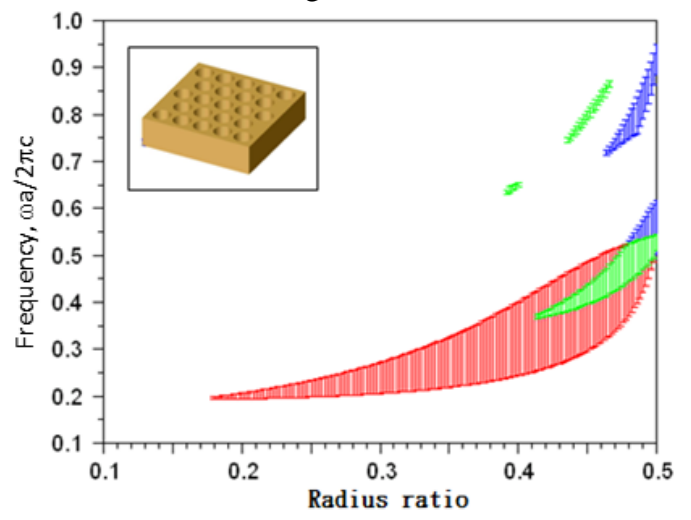

Fig. 5. Band gap diagram for a two-dimensional triangular lattice array based on air holes in silicon slab. TE mode in blue, TM mode in red, and complete band gaps in green

For the two-dimensional beam-splitter, the structure has two orthogonal line defects and an inserted point defect. It has two input ports and two output ports, as is depicted in Fig. 6. The wave propagation has been analyzed by the FDTD 2-dimensional method while one of the input light energy could be divided into the two output ports with the inserted point defect.

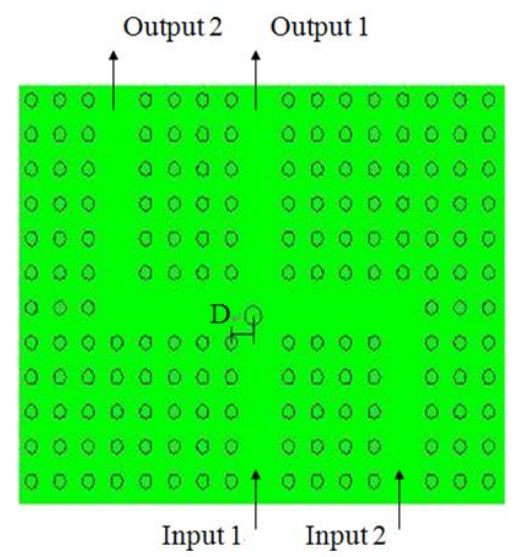

Fig. 6. The beam-splitter structure with a point defect inserted in the crossed line defects

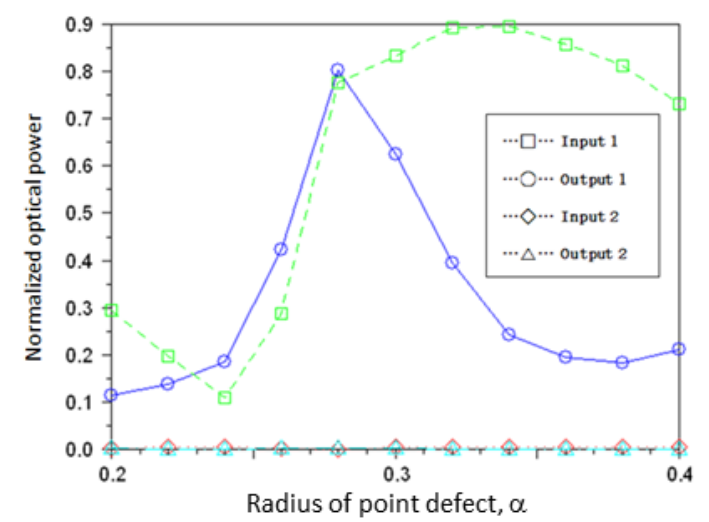

Fig. 7. The normalized optical power of the two input ports and the two output ports

The frequency was chosen based on the band gap range of 0.2 to 0.4 radius ratio for square lattice array based on silicon pillars. At first, we launched a light energy into the input port1, then monitored the input and output port power 
levels. The normalized optical power results, or the ratios between the measured and launched powers, thus dimensionless, are shown in Fig. 7.

The point defect radius has been varied in the center of the cross orthogonal line defects. The point defect radius controlled the transmission and reflection of light. When the input light encountered the point defect, the magnetic wave either passed through or became reflected to reduce the power. The point defect radius thus affected the photonic band for a specific wavelength. It has been found that output port one power would equal input port one at the radius of 0.28 a.

It was also desired to divide any input light energy equally to the two output ports by simply moving the point defect to the suitable position. Fig. 8, therefore, depicts the normalized optical power of the four ports when moving the point defect for a distance of 0.6 a to 0.9 a from the marked cell D in Fig. 6.

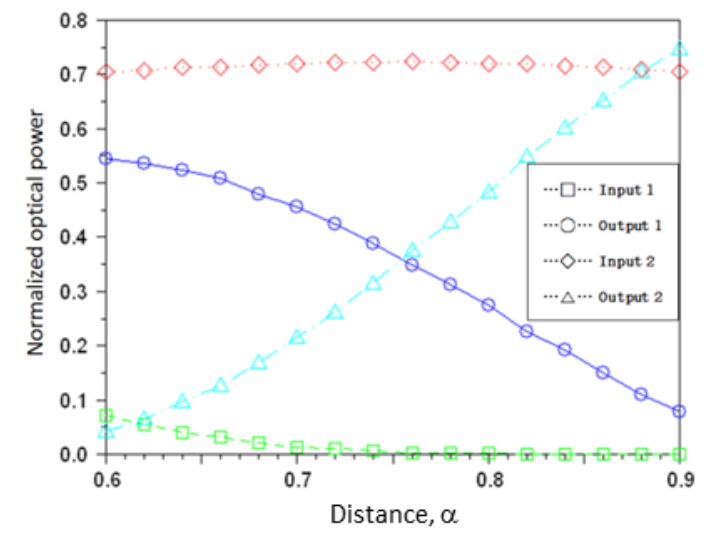

Fig. 8. The effects of moving the central point defect for a distance on the normalized optical power of the four ports

The polarization was perpendicular to the propagation plane. The best distance was identified to be $0.76 \mathrm{a}$. By moving the point defect, we could change the directions of the input wave coupling to comply with a specific requirement. On the other hand, by launching two lights of different phases into the two input ports, the output ports could exhibit Mach-Zehnder interferometer beam splitter results, as shown in Fig. 9.

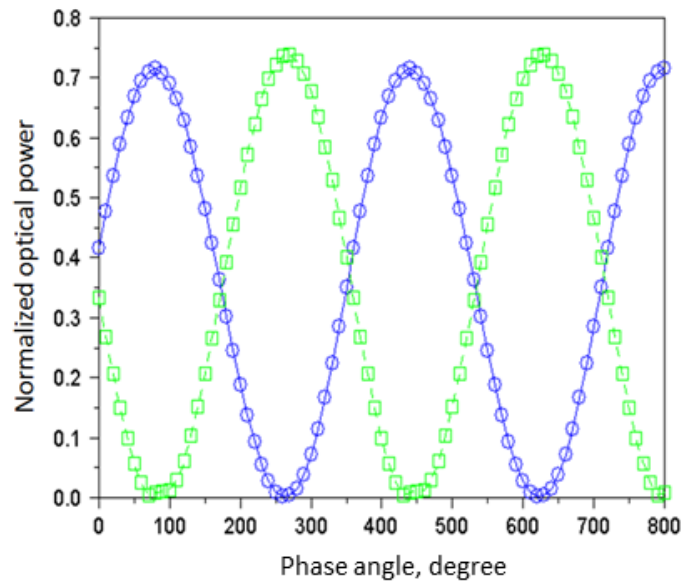

Fig. 9. Output power versus phase angle difference to verify Mach-Zehnder interferometer characteristics

When the two imported lights had a phase difference of $90^{\circ}$, the waves would have interfered in the designated point defect. Also, the silicon based beam-splitter could be defined by an e-beam writer and using the well-developed semiconductor processing technology.

\section{CONCLUSIONS}

In summary, we have investigated the photonic crystal band gap structures for two-dimensional beam splitters. They could exhibit the Mach-Zehnder interferometer characteristics, and showed great potential applications to optical switches and optical integrators. The input port one power of the silicon device would equal the output port one at the defect radius of 0.28 a while a was defined as the lattice constant of the photonic crystal structure. Also, it has been desired to divide the input light energy equally to the two output ports by moving the designated point defect. The best moving distance was also identified at $0.76 \mathrm{a}$.

\section{Acknowledgement}

This work was supported in part by the Ministry of Science and Technology under research grant MOST1042221-E182-073 (BMRP246).

\section{REFERENCES}

1. Yablonovitch, E. Photonic Crystals: Semiconductors of Light Scientific American 285 2001: pp. 34-41.

2. Yeng, Y.X., Chan, W.R., Rinnerbauer, V., Stelmakh, V., Senkevich, J.J., Joannopoulos, J.D., Soljai, M., Elanovi, I. Photonic Crystal Enhanced Silicon Cell Based Thermophotovoltaic Systems Optics Express 23 2015: pp. A157-A168. http://dx.doi.org/10.1364/OE.23.00A157

3. Wu, G.M., Tsai, B.H., Kung, S.F., Wu, C.F. Improved Light Extraction Efficiency by Photonic Crystal Arrays on Transparent Contact Layer Using Focused Ion Beams Acta Physica Polonica A 120 2011: pp. 140-143.

4. Wu, Z.H., Zhang, Q., Tang, B., Qiu, Y., Luo, Q. Optical Switch Based on the Liquid-Crystal-Modulated SelfCollimating Photonic Crystals Materials Research Innovations 19(S1) 2015: pp. 219-221. http://dx.doi.org/10.1179/1432891715z.0000000001409

5. Oates, A., Cabrera-Espana, F.J., Agrawal, A., Reehal, H.S. Fabrication and Characterisation of $\mathrm{Si}$ Micropillar PV Structure Materials Research Innovations 18 2014: pp. 500-504. http://dx.doi.org/10.1179/1433075X14Y.0000000244

6. Hu, Y., Liu, G.Q., Zhang, X.N., Cai, Z.J. A New TwoDimensional Photonic Crystal Channel Drop Filter Based on Two-Resonant Cavities Advanced Materials Research 760 2013: pp. 397-400. http://dx.doi.org/10.4028/www.scientific.net/AMR.760-762.397

7. Han, T., Liu, Y.G., Wang, Z., Guo, J., Wu, Z., Luo, M., Li, S., Wang, J., Wang, W. Control and Design of Fiber Birefringence Characteristics Based on Selective-Filled Hybrid Photonic Crystal Fibers Optics Express 22 2014: pp. $15002-15016$. http://dx.doi.org/10.1364/OE.22.015002

8. Wu, L. J., Xu, Z.J., Shen, L.H., Han, Y. Negative Differential Conductivity and Reversal Spin-Polarised Current Through Quantum-Dot Ring Embedded in Aharonov-Bohm Interferometer Materials Research Innovations 18 (S4) 2014: pp. 1045-1051. http://dx.doi.org/10.1179/1432891714z.000000000919 
9. Furumi, S. Self-Assembled Organic and Polymer Photonic Crystals for Laser Applications Polymer Journal 45 2013: pp. 579-593.

10. Lu, Y.H., Liu, K.J., Wu, G.M. Top-Contact Organic ThinFilm Transistors with Improved Mobility and Turn-On Voltage Materials Research Innovations 19 (S5) 2015: pp. $1344-1347$. http://dx.doi.org/10.1179/1432891714z.0000000001308

11. Umenyi, A.V., Kawashiri, S.Y., Miura, K., Hanaizumi, O. Theoretical Analysis of Photonic Band Gaps and Defect Modes of Novel Photonic Crystal Waveguides Consisting of Si-Ion Implanted $\mathrm{SiO}_{2}$ Using the Finite-Difference TimeDomain Method Key Engineering Materials 459 2011: pp. $162-167$. http://dx.doi.org/10.4028/www.scientific.net/KEM.459.162

12. Patil, P.B., Pendharker, S., Shevgaonkar, R.K. Electrical Modeling of Photonic Crystal Defects Microwave and Optical Technology Letters 54 2012: pp. 2522-2528. http://dx.doi.org/10.1002/mop.27115

13. Lu, R.C., Wang, C.M., Lee, K.Y. Characteristic of Point Defect Around the Photonic Crystal Bend Advanced Materials Research 571 2012: pp. 300-303.

14. Hamidi, S.M., Tehranchi, M.M., Shasti, M. Engineered One-Dimensional Magneto-Photonic Crystals for Wavelength Division Multiplexing Systems Journal of Physics D-Applied Physics 44 2011: pp. 205107-1-205107-5.

15. Kumar, V., Suthar, B., Kumar, A., Singh, K.S., Bhargava, A. Design of A Wavelength Division Demultiplexer Using Si-Based One-Dimensional Photonic Crystal with a Defect Optik 124 2013: pp. 2527-2530.

16. Jons, J.D., Rengstl, U., Oster, M., Hargart, F., Heldmaier, M., Bounouar, S., Ulrich, S.M., Jetter, M., Michler, P. Monolithic On-Chip Integration of Semiconductor Waveguides, Beamsplitters and SinglePhoton Sources Journal of Physics D-Applied Physics 48 2015: pp. 085101-1-085101-6.

17. Ishizaki, K., Koumura, M., Suzuki, K., Gondaira, K., Noda, S. Realization of Three-Dimensional Guiding of
Photons in Photonic Crystals Nature Photonics 2013: pp. $133-137$.

18. Lee, K.Y., Wu, G.J., Lin, H.L., Liu, C.K. An Interesting Optical Behavior of Bent/Multi-Branch Waveguides Based on Hexagonal-Lattice Photonic Crystals Journal of Optical Communication 32 2011: pp. 225-228.

19. Takeda, H., Sakoda, K. Bending Losses of Optically Anisotropic Exciton Polaritons in Organic Molecular-Crystal Nanofibers Optics Express 21 2013: pp. 31420-31429. http://dx.doi.org/10.1364/OE.21.031420

20. Chantakit, T., Srinuanjan, K., Yupapin, P.P. Two Dimension Photonic Crystal Y-Branch Beam Splitter with Variation of Splitting Ratio Based on Hybrid Defect Controlled Applied Physics A-Materials Science \& Processing 117 2014: pp. 547-552.

21. Chen, C.C., Chien, H.D., Luan, P.G. Photonic Crystal Beam Splitters Applied Optics 43 2004: pp. 6187-6190. http://dx.doi.org/10.1364/AO.43.006187

22. Lourtioz, J.M., Benisty, H., Berger, V., Gerard, J.M., Maystre, D., Tchelnokov, A. Photonic CrystalsTowards Nanoscale Photonic Devices, 2nd edn, Springer, BerlinHeidelberg, 2008: pp. 80-104.

23. Poborchii, V., Tada, T., Morita, Y., Kanayama, T. Ultraviolet Raman Spectra of Single Uncoated and SiO2Coated Silicon-on-Insulator Nanowires: Phonon Boundary Scattering, Wave-Vector Relaxation and Stress Applied Physics Letters 103 2013: pp. 153104-1-153104-4.

24. Fan, Z., Liu, J., Chen, S., Chang, H., Guan, C., Yuan, L. Comparative Study of Photonic Band Gaps of GermaniumBased Two-Dimensional Triangular-Lattice and SquareLattice and Decagonal Quasi-Periodic Photonic Crystals Microelectronic Engineering 96 2012: pp. 11-17. http://dx.doi.org/10.1016/j.mee.2012.02.041

25. Zhang, K., Du, X., Katz, M.B., Li, B., Kim, S.J., Song, K., Graham, G.W., Pan, X. Creating High Quality Ca:TiO ${ }_{2}-\mathrm{B}$ $\left(\mathrm{CaTi}_{5} \mathrm{O}_{11}\right)$ and $\mathrm{TiO}_{2}-\mathrm{B}$ Epitaxial Thin Films by Pulsed Laser Deposition Chemical Communications 51 2015: pp. $8584-8587$. 